\title{
Pituitary volume in psychosis
}

\author{
CARMINE M. PARIANTE, KONSTANTINA VASSILOPOULOU, \\ DENNIS VELAKOULIS, LISA PHILLIPS, BRIDGET SOULSBY, \\ STEPHEN J. WOOD, WARRICK BREWER, DEIDRE J. SMITH, PAOLA DAZZAN, \\ ALISON R. YUNG, IOANNIS M. ZERVAS, GEORGE N. CHRISTODOULOU, \\ ROBIN MURRAY, PATRICK D. MCGORRY and CHRISTOS PANTELIS
}

\begin{abstract}
Background Patients with psychosis have activation of the hypothalamicpituitary-adrenal (HPA) axis during the acute phase of the psychosis. Whether this has any morphological consequences for the pituitary gland is currently unknown.
\end{abstract}

Aims To examine pituitary volume variation in people at different stages of psychotic disorder.

Method Pituitary volume was measured using $1.5 \mathrm{~mm}$, coronal magnetic resonance images in 24 people with firstepisode psychosis, 51 with established schizophrenia and 59 healthy controls.

Results Compared with the control group, the people with first-episode psychosis had pituitary volumes that were 10\% larger, whereas those with established schizophrenia had pituitary volumes that were $17 \%$ smaller. In both of the groups with psychosis, there was no difference in pituitary volume between those receiving typical antipsychotic drugs and those receiving atypical antipsychotics.

Conclusions The first episode of a psychosis is associated with a larger pituitary volume, which we suggest is due to activation of the HPA axis. The smaller pituitary volume in the group with established schizophrenia could be the consequence of repeated episodes of HPA axis hyperactivity.

Declaration of interest None. Funding detailed in Acknowledgements.
'The period of acute psychotic turmoil (or panic, or ego disintegration), which initiated the psychotic episodes,.... was shown to be associated with very great elevations in corticosteroid. . . excretion' (Sachar et al, 1970).

People who are in the acute phase of a psychotic disorder, with florid symptoms, newly hospitalised or unmedicated, show hypothalamic-pituitary-adrenal (HPA) axis hyperactivity, but the central mechanisms underlying this neuroendocrine abnormality are unclear (Cotter \& Pariante, 2002). In major depression, HPA axis hyperactivity has been associated with an increased volume of the pituitary gland measured using magnetic resonance imaging (MRI), which correlates with the circulating cortisol levels (Krishnan et al, 1991; Axelson et al, 1992). To investigate whether HPA axis activation in psychosis is also associated with increased pituitary volume, we measured the pituitary glands of patients experiencing their first episode of psychosis, when they are most likely to show HPA axis abnormalities. We also measured pituitary volumes in a group of people with established schizophrenia, to investigate whether this putative abnormality varies at different stages of the psychotic disorder.

\section{METHOD}

\section{Participants}

We studied 134 individuals: 24 people with first-episode psychosis, 51 with established schizophrenia and 59 healthy controls. These participants received a brain MRI scan as part of a large study investigating brain structural abnormalities in psychosis (Velakoulis et al, 1999). All were screened for comorbid medical and psychiatric conditions by clinical assessment and by physical and neurological examination. Exclusion criteria were a history of significant head injury or seizures, electroconvulsive therapy during the 6 months before the scan, polydipsia, neurological disease, impaired thyroid function, asthma, diabetes, steroid use or meeting DSM-IV (American Psychiatric Association, 1994) criteria for alcohol or substance abuse or dependence. Individuals with a metallic object in the body or other contraindication to MRI, and control volunteers with a personal or family history of psychiatric illness, were also excluded. After complete description of the study, written informed consent was obtained from all participants. The local Internal Review Board (Research and Ethics Committee) approved the study.

\section{First-episode group}

Twenty-four in-patients with first-episode psychosis were recruited from the Early Psychosis Prevention and Intervention Centre (EPPIC) in Melbourne, Australia (McGorry et al, 1996). Study inclusion criteria were age at onset 16-30 years and current psychosis, reflected by the presence of at least one of delusions, hallucinations, disorder of thinking or speech (other than simple accelerations or retardation) or disorganised, bizarre or markedly inappropriate behaviour. Patients' DSM-IV diagnoses were based on chart review, the Structured Clinical Interview for DSM-IV Disorders (SCID; First et al, 1997) and the Royal Park Multidiagnostic Instrument for Psychosis (RPMIP; McGorry et al, 1989), administered during the initial treatment episode. Eleven patients had a diagnosis of schizophrenia or schizophreniform disorder, 9 of schizoaffective disorder, 2 of bipolar disorder with psychotic symptoms, 1 of psychosis not otherwise specified and 1 of delusional disorder. Members of this group were antipsychotic-naïve before their admission. At the time of the scan, 23 out of 24 patients were taking antipsychotic medication: 14 were receiving a typical antipsychotic agent (mean daily dosage $153 \mathrm{mg}$ (s.d.=81) chlorpromazine equivalents) and 9 an atypical agent, of whom 7 were receiving risperidone (mean daily dosage $2.9 \mathrm{mg}$ (s.d.=1.5)), 1 olanzapine and 1 clozapine. Thirteen patients were taking benzodiazepines, 6 were taking an anticholinergic, 4 lithium and 2 an antidepressant. We collected sociodemographic and clinical information including the date of first admission, medication data and level of compliance. 


\section{Established schizophrenia group}

Fifty-one people with established schizophrenia were recruited from the rehabilitation unit of the Royal Park Hospital in Melbourne, Australia. Diagnoses were based on clinical and chart review using DSM-III-R criteria (American Psychiatric Association, 1987). All of these patients had been ill for at least 5 years from the time of their first admission. Of the 42 patients for whom complete medication data were available, 39 were receiving antipsychotic treatment at the time of scanning: 19 were taking a typical antipsychotic agent (mean daily dosage $682 \mathrm{mg}$ (s.d.= 554) chlorpromazine equivalents - a significantly higher dosage than that in the group with first-episode psychosis: analysis of variance, $F=12.5$, d.f. $=1,31 ; P=0.001)$ and 20 were taking an atypical antipsychotic, of whom 16 were receiving clozapine (mean daily dosage $450 \mathrm{mg}$ (s.d.=225)), 2 olanzapine, 1 quetiapine and 1 risperidone. Sixteen patients were taking benzodiazepines, 8 were taking an anticholinergic, 6 an antidepressant and 5 lithium. We collected socio-demographic and clinical information including the date of first admission, medication data and level of compliance.

\section{Control group}

Fifty-nine healthy volunteers were recruited by approaching ancillary hospital staff and through advertisements. They were drawn from socio-demographic backgrounds similar to those of the participants with psychotic disorders.

\section{Scanning and data analysis}

All participants were scanned using a GE Sigma 1.5 T scanner (GE Medical Systems, Milwaukee, USA) at the Royal Melbourne Hospital. Head movement was minimised by the use of foam padding and restraining straps across the forehead and chin. All patients received their normal medication on the day of scanning. A three-dimensional volumetric spoiled gradient recalled echo in the steady state sequence generated 124 contiguous $1.5 \mathrm{~mm}$ coronal slices. Imaging parameters were time to echo $(3.3 \mathrm{~ms}$, time to repetition $\left(14.3 \mathrm{~ms}\right.$, flip angle $30^{\circ}$, matrix size $256 \times 256$, field of view $24 \mathrm{~cm} \times 24 \mathrm{~cm}$ and voxel dimension $0.938 \mathrm{~mm} \times 0.938 \mathrm{~mm} \times 1.5 \mathrm{~mm}$. The scanner was calibrated fortnightly using the same proprietary phantom to ensure stability and accuracy of measurements.
Imaging data were transferred from digital audiotape to an SGI O2 workstation (SGI, Mountain View, California, USA) and coded to ensure patient confidentiality and masked rating of data. All volumes were estimated using ANALYZE 7.5 (Mayo Clinic). Methods for estimating whole brain volume and intracranial volume have been described by Velakoulis et al (1999) and Eritaia et al (2000). Whole brain volume includes the hemispheres, cerebellum, brain-stem and the ventricles, but not the cisterns or sulcal cerebrospinal fluid. Intracranial volume represents the space within the following boundaries: dura mater, the undersurfaces of the frontal lobe, the dorsum sellae, the clivus and, at the craniovertebral junction, the attachment of the dura to the posterior, cutting across to the anterior arch of $\mathrm{C} 1$. Interrater and intrarater reliabilities were 0.99 for both measurements.

\section{Pituitary measurement}

Each pituitary gland was traced in all coronal slices where it could be visualised, using a method described by Sassi et al (2001). The mean number of coronal slices traced per case was 13 (range 9-16). The pituitary stalk was excluded from the tracings, but we included a posterior bright spot, corresponding to the posterior pituitary, the intensity of which is thought to reflect vasopressin concentrations (Sassi et al, 2001). We traced around the usually well-defined borders of the anterior and posterior pituitary: the diaphragma sellae, superiorly; the sphenoid sinus, inferiorly; and the cavernous sinuses, bilaterally (Fig. 1) (Lurie et al, 1990; Elster, 1993; Lum et al, 2002). Volume of the pituitary (in $\mathrm{mm}^{3}$ ) was calculated by summing volumes for all relevant slices. One rater (K.V.) traced all the pituitaries in the study. Before starting the study, she trained with a senior author involved in the development and optimisation of the technique (B.S.). The intrarater and interrater reliabilities were calculated by measuring the pituitary volumes in ten scans randomly selected from the original pool of MRI scans from all the study groups. The interrater reliability was 0.85 , the intrarater reliability was 0.97 and the average variation between two measurements of the same pituitary was $5 \%$.

\section{Statistical analysis}

Original data are presented as mean and standard deviation. Adjusted means are presented as mean and standard error of the mean (s.e.m.). Clinical and sociodemographic differences between groups were examined using one-way analysis of variance (ANOVA) followed by post hoc Student-Newman-Keuls test (Table 1). To limit the number of statistical comparisons, the differences in pituitary volumes between the control group and the two clinical groups were examined by conducting a single two-way analysis of covariance (ANCOVA) test, using the intracranial volume as a covariate, followed by pairwise comparisons of estimated means. By
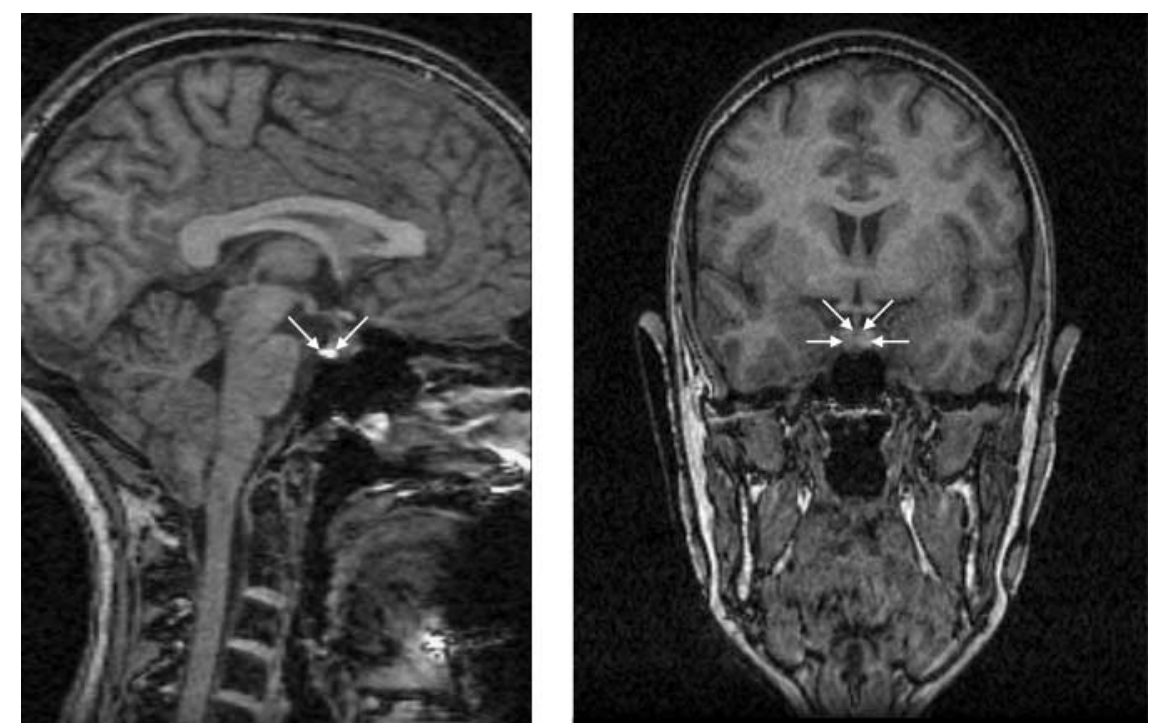

Fig. I Pituitary gland (arrowed) viewed in sagittal (left) and coronal (right) magnetic resonance imaging scans. Coronal slices were used for tracing the gland. 


\begin{tabular}{|c|c|c|c|c|}
\hline & $\begin{array}{l}\text { Control group } \\
\qquad(n=59)\end{array}$ & $\begin{array}{l}\text { First-episode } \\
\text { group }(n=24)\end{array}$ & $\begin{array}{l}\text { Established schizophrenia } \\
\qquad \text { group }(n=51)\end{array}$ & Group comparisons \\
\hline Age, years: mean (s.d.) & $29.3(10.5)$ & $21.9(3.3)$ & $41.0(9.3)$ & ANOVA, $F=4 \mid .2$, d.f. $=2,|3| ; P<0.00 \mathrm{I}$ \\
\hline \multicolumn{5}{|l|}{ Gender } \\
\hline Male/female, $n / n$ & $33 / 26$ & $18 / 6$ & $41 / 10$ & $\chi^{2}=8.2$, d.f. $=2, P=0.017$ \\
\hline Male, \% & $56 \%$ & $75 \%$ & $80 \%$ & \\
\hline Age at first admission, years: mean (s.d.) & & $21.8(3.2)$ & $22.1(7.2)$ & ANOVA, $F=0.03$, d.f. $=I, 73 ; P=0.97$ \\
\hline Time after first admission, years: mean (s.d.) & & $0.2(0.4)$ & $19.1(10.2)$ & ANOVA, $F=40.4$, d.f. $=1,73 ; P=0.00 \mathrm{I}$ \\
\hline Intracranial volume, $\mathrm{mm}^{3}$ : mean (s.d.) & | 44220 | (142599) & | 407 | 97 ( |37 |36) & I 449456 (|142599) & ANOVA, $F=0.8$, d.f. $=2,|3| ; P=0.5$ \\
\hline Whole brain volume, $\mathrm{mm}^{3}$ : mean (s.d.) & I $349542(150004)$ & 1349440 (14462I) & 1318138 (125094) & ANOVA, $F=0.8$, d.f. $=2,|3| ; P=0.5$ \\
\hline
\end{tabular}

ANOVA, analysis of variance.

definition, it was impossible to have a single control group that was comparable with the young first-episode group as well as the older group with established schizophrenia, for both age and gender distribution. Therefore, to control for these potential confounders, gender was used as betweensubject factor, and age (and whole brain volume) were included as covariates in a second set of confirmatory analyses. We have previously used this approach to demonstrate differences in hippocampal volumes between normal volunteers, people with a first psychotic episode and people with established schizophrenia (Velakoulis et al, 1999). Moreover, the results obtained from this analysis were corroborated by conducting two separate ANCOVA tests comparing each of the clinical groups with another control group that had a similar age and gender distribution. These two control subgroups were obtained from the original control sample by ranking males and females by age (masked to their pituitary volume) and excluding older controls (in the comparison with the first-episode group) or younger controls (in the comparison with the established schizophrenia group) until comparable age and gender distributions were achieved (see Results). The ANCOVA test was also used to compare pituitary volumes between the following subgroups:

(a) males and females, across the whole sample;

(b) first-episode patients with schizophrenia or schizophreniform psychosis, first-episode patients with other diagnoses, and controls; (c) patients receiving typical antipsychotics and patients receiving atypical antipsychotics;

(d) patients receiving lithium and/or antidepressants and patients not receiving these drugs.

Partial correlations, covarying for intracranial volume, were conducted between pituitary volume and age.

\section{RESULTS}

\section{Characteristics of the sample}

The main demographic and clinical features of the three groups are presented in Table 1. As expected, there were significant differences in age and gender between them. Post hoc comparisons showed that the control group members were younger than the patients with established schizophrenia and older than the patients with first-episode psychosis, and that there were significantly more males in the established schizophrenia group than in the control group. By definition, patients with established schizophrenia had a much longer duration of illness at the time of the MRI scan than the first-episode group. Although there was some variability in the duration of illness at the time of the scan in the firstepisode group, 21 out of 24 of these patients were scanned within 2 months of their first admission. Intracranial volume and whole brain volume did not differ between the groups to a statistically significant extent.

\section{Pituitary volume}

There was a significant difference in pituitary volume between the three groups (ANCOVA, $F=18.0$, d.f. $=2,132 ; P<0.001$ ). Figure 2 shows the pituitary volumes of

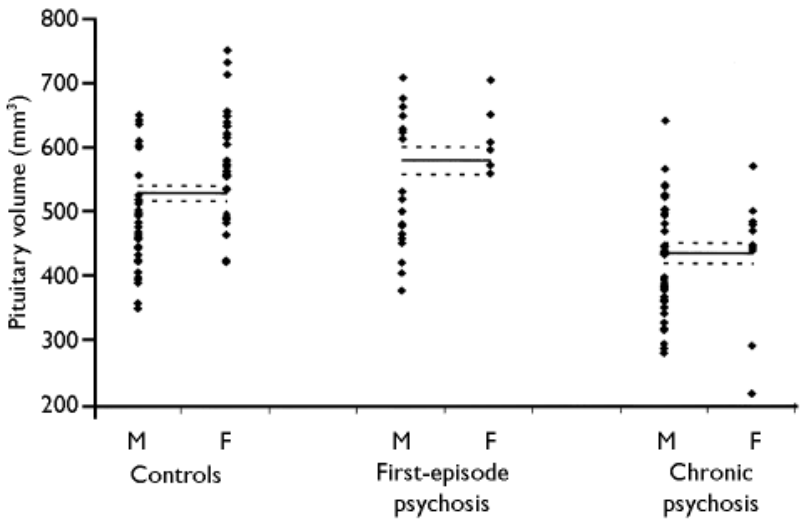

Fig. 2 Pituitary volumes in participants with first-episode psychosis, participants with established schizophrenia (chronic psychosis) and in healthy volunteers (controls). Each group is divided into males (M) and females (F). The figure also shows the estimated mean and standard error of the mean values for each group (controls, first-episode and chronic) after adjustment for intracranial volume and gender. 
all participants, and the estimated mean and s.e.m. values for each group after adjustment for intracranial volume and gender. Although examination of the individual data showed an overlap between the groups, the presence of group differences was suggested by the fact that 17 of the 24 patients in the first-episode group $(71 \%)$ had pituitary volumes that were larger than the median of the control group, whereas 35 of the 51 patients in the established disease group (69\%) had pituitary volumes that were smaller than the median of the control group. The ANCOVA analysis showed that pituitary volumes in the first-episode group were, on average, $10 \%$ larger than those of the control group $\left(+52 \mathrm{~mm}^{3}\right.$, s.d. $\left.=24, \quad P=0.032\right)$, whereas pituitary volumes in the group with established schizophrenia were, on average, $17 \%$ smaller than controls $\left(-91 \mathrm{~mm}^{3}\right.$, s.d. $=19, P<0.001)$. These differences remained significant when age and whole brain volume were used as covariates in the analysis. Furthermore, these differences remained significant when each clinical group was compared with a control group that had similar age and gender distribution (obtained as described in the statistical analysis section, above; 32 controls in the comparison with the first-episode group and 29 controls in the comparison with the established illness group; ANOVA for age differences, $F<0.2, P>0.6$, for both comparisons; chi-squared test for gender differences, $\chi^{2}<2.1, P>0.2$, for both comparisons).

In the whole sample, women had larger pituitaries than men: $553 \mathrm{~mm}^{3}($ s.d. $=17) v$. $484 \mathrm{~mm}^{3}$ (s.d.=10); ANCOVA, $F=11.7$, d.f. $=1,133 ; P=0.001$. There was a trend for a gender $\times$ group interaction (ANOVA, $F=2.5$, d.f. $=2,132 ; P=0.085$ ), which was due to a smaller gender effect in the group with established schizophrenia in comparison with the other groups (Fig. 2). In the whole sample, there was a negative correlation between age and pituitary volume $(r=-0.36$, d.f. $=131 ; P<0.001)$, present in both males $(r=-0.26$, d.f. $=89$, $P=0.012)$ and females $(r=-0.56$, d.f. $=39$; $P<0.001)$

\section{Effects of clinical characteristics and subgroups}

In the first-episode group, both patients with schizophrenia or schizophreniform psychosis $(n=11)$ and patients with other diagnoses $(n=13)$ had larger pituitary volumes than the control group; this subgroup analysis, however, did not reach statistical significance (ANCOVA, $F=1.9$, d.f. $=2,54 ; \quad P=0.17$; schizophrenia/schizophreniform psychosis $+48 \mathrm{~mm}^{3} \quad($ s.d. $=30)$ $v$. controls, $P=0.12$; other diagnoses $+50 \mathrm{~mm}^{3}$ (s.d. $\left.=36\right) v$. controls, $P=0.16$ )

There was no difference in pituitary volume between patients receiving typical and atypical antipsychotics, either in the first-episode group (ANCOVA, $F=1.6$, d.f. $=1,22 ; P=0.2)$ or in the established schizophrenia group (ANCOVA, $F=1.3$, d.f. $=1,38 ; P=0.3)$. Similarly, there was no difference in pituitary volume between the patients receiving antidepressants or lithium and the other patients, either in the first-episode group (ANCOVA, $F<0.1$, d.f. $=1,23 ; P=0.97)$ or in the established illness group (ANCOVA, $F=0.5$, d.f. $=1,41 ; P=0.5$ ).

\section{DISCUSSION}

The pituitary gland regulates HPA axis activity by secreting adrenocorticotrophic hormone, which in turn stimulates the adrenal gland to produce cortisol. We found that patients with a first episode of psychosis had larger pituitary volumes than healthy volunteers, whereas patients with established schizophrenia (of at least 5 years' duration) had smaller pituitary volumes than the controls.

\section{Mechanisms leading to increased pituitary volume}

We suggest that the increased pituitary volume in patients with first-episode psychosis is due to activation of the HPA axis and, in particular, to an increase in the size and number of corticotrophs (cells producing adrenocorticotrophic hormone). The few studies that have examined the HPA axis in people with a first episode of psychosis have found hyperactivity of this hormonal system (Sachar et al, 1970; Ryan et al, 2003). Interestingly, both HPA axis hyperactivity and increased pituitary volume have been described in patients with severe major depression (Krishnan et al, 1991; Axelson et al, 1992), and have been interpreted in these patients as showing a lack of negative inhibitory feedback by circulating glucocorticoid hormones on the HPA axis, especially at the level of the pituitary (glucocorticoid resistance) (Pariante \& Miller, 2001; Pariante, 2003). Indeed, increased size and number of corticotrophs and increased pituitary volume are present also in people with a lack of negative inhibitory feedback by circulating glucocorticoid hormones due to Addison's disease (Mineura et al, 1987). Therefore, our findings suggest that glucocorticoid resistance may be present in people with first-episode psychosis. As glucocorticoid resistance is a common correlate of stress-induced HPA axis activation in animals and humans (Raison $\&$ Miller, 2003), our findings could be explained by an activation of the stress response. Such activation could be due to the distress caused by the first psychotic experience, to an increased biological susceptibility to daily life stress or to an increased level of independent stressors leading to the psychotic episode - or to all these causes (Sachar et al, 1970; Bebbington et al, 1993; Myin-Germeys et al, 2001). Reassuringly, there is no evidence that HPA axis hyperactivity causes damage to the brain in people with mental disorders (Muller et al, 2001). We did not measure hormonal levels in our study participants, however, so our proposed relationship between pituitary hyperplasia and hyperactivity of the HPA axis remains speculative.

\section{Mechanisms leading to decreased pituitary volume}

The second major finding of our study was that the group of people with established schizophrenia had smaller pituitary volumes than the control group. This finding is remarkably consistent with other studies, which have shown reduced pituitary volumes in patients with an eating disorder (Doraiswamy et al, 1990) and in euthymic and depressed patients with bipolar disorder (Sassi et al, 2001). Therefore, it is possible that common pathological mechanisms are present in people with mental disorders that lead to hypoplasia of the pituitary gland. Interestingly, based on the evidence that patients with bipolar disorder can present with HPA axis hyperactivity, Sassi et al (2001) have suggested that chronic activation of the HPA axis might decrease pituitary volume by reducing (through negative feedback) the function of cells producing other pituitary hormones. Because psychotic relapses are associated with activation of the HPA axis (Tandon et al, 1991), it is possible that repeated episodes of HPA axis activation associated with previous relapses in our group of patients with established schizophrenia led 
to the reductions in pituitary volume. Again, without having measured pituitary hormones, we do not know whether this volume reduction has any functional consequences. Previous studies have found normal HPA axis function in people with established psychosis, especially if they were clinically stable and receiving treatment (Tandon et al, 1991).

This is a cross-sectional study comparing different people at various stages of psychosis, not a prospective study; therefore, we can only speculate that the people in the first-episode group with large pituitary glands would 'progress' to having small pituitary glands 5 years later. It is also possible that these two groups are biologically distinct. The patients with established schizophrenia might have had a smaller pituitary volume even at the onset of their psychosis, for example, because of a neurodevelopmental problem (Lum et al, 2002). Equally, patients with first-episode psychosis might have a normal pituitary volume if scanned in the future, as found in people with major depression in the euthymic phase (Sassi et al, 2001).

\section{Limitations of the study}

As already stated, we did not measure hormonal levels in these samples. Moreover, the neuroimaging data were initially collected to examine brain structure, rather than neuroendocrine abnormalities. Future studies on this topic should include hormone measurements. A possible limitation of our study, common to all studies examining pituitary volume by imaging methods, is the difficulty in distinguishing between anterior and posterior pituitary volumes (Doraiswamy et al, 1990; Lurie et al, 1990; Krishnan et al, 1991; Axelson et al, 1992; Sassi et al, 2001). However, the posterior pituitary, which releases vasopressin and oxytocin, constitutes less than $20 \%$ of the total pituitary volume and in contrast to the anterior pituitary - there is no known condition associated with its enlargement, except tumour (Krishnan et al, 1991; Elster, 1993). Therefore, we believe that the changes in volume we have described are due to changes in the volume of the anterior pituitary.

Finally, we cannot exclude that pituitary hyperplasia in first-episode psychosis is due to increased function of pituitary cells secreting hormones other than adrenocorticotrophic hormone, such as growth hormone and prolactin; levels of these hormones are also elevated by stress. Furthermore, the patients in our firstepisode group were receiving neuroleptic treatment, and antipsychotic drugs can induce proliferation of prolactin-secreting cells in animals (Saiardi et al, 1997). However, in these patients pituitary volume did not seem to be univocally correlated with antipsychotic administration. In fact, patients in both the first-episode group and the established schizophrenia group were receiving antipsychotic medication, but the differences in pituitary volume, compared with controls, were in opposite directions; moreover, there was no difference in pituitary volume between patients taking typical and atypical antipsychotics, even though the effects of these two kinds of drugs on prolactin secretion are quite different (Halbreich \& Kahn, 2003).

\section{Strengths of the study}

Three lines of evidence support our conclusions. First, the effects of age and gender on pituitary volume in our sample are consistent with previously published studies (Lurie et al, 1990; Sassi et al, 2001). Second, the most common causes of increased pituitary volume - administration of exogenous oestrogens, hypothalamic tumour, pregnancy, primary hypothyroidism and puberty (Elster, 1993) - were excluded in our study sample. Third, these results are not limited by the socio-demographic differences between the three groups, because variables that could regulate pituitary volumes (age, gender, intracranial volume and whole brain volume) are controlled, both in the main statistical analysis and in the two direct comparisons between each clinical group and the matched control groups.

Although the fact that the first-episode sample was clinically heterogeneous could be interpreted as a limitation, studies on such individuals usually include all diagnoses of psychosis. In fact, because of the temporal instability of the diagnosis in these patients, restricting the analysis to a single diagnostic group in the initial stages would imply missing or misdiagnosing a large number of individuals who would have qualified at a later stage (Amin et al, 1999). Future studies on similar, larger samples would clarify whether there are differences in pituitary volume across psychiatric diagnoses. Moreover, prospective studies of HPA axis function and brain imaging in people at various stages of psychosis, including those at high risk of developing psychosis, will clarify whether these pituitary abnormalities are indeed related to HPA axis function and whether measurements of the stress response could be used to predict the development of psychosis.

\section{ACKNOWLEDGEMENTS}

This research was funded by project grants from the National Health and Medical Research Council grant numbers 970598, 970391 and 981112, the Victorian Health Promotion Foundation, the Stanley Foundation, the lan Potter Foundation, the Woods Family Trust, the Australian Communications and Computing Institute, the Percy Baxter Charitable Trust and the National Alliance for Research on Schizophrenia and Depression (NARSAD). C.M.P. was funded by a travel award from the Wellcome Trust and two travel grants from Brain. P.D. was funded by a short-term travel grant from the Wellcome Trust and a grant from the University of London. C.M.P. and P.D. have both received a NARSAD Young Investigator Award for their research in the UK.

\section{REFERENCES}

American Psychiatric Association (1987) Diagnostic and Statistical Manual of Mental Disorders (3rd edn, revised) (DSM-III-R). Washington, DC: APA.

American Psychiatric Association (1994) Diagnostic and Statistical Manual of Mental Disorders (4th edn) (DSM-IV). Washington, DC: APA.

Amin, S., Singh, S. P., Brewin, J., et al (1999) Diagnostic stability of first-episode psychosis. Comparison of ICD-10 and DSM-III-R systems. British Journal of Psychiatry, 175, 537-543.

Axelson, D. A., Doraiswamy, P. M., Boyko, O. B., et al (1992) In vivo assessment of pituitary volume with magnetic resonance imaging and systematic stereology: relationship to dexamethasone suppression test results in patients. Psychiatry Research, 44, 63-70.

Bebbington, P., Wilkins, S., Jones, P., et al (1993) Life events and psychosis. Initial results from the Camberwell Collaborative Psychosis Study. British Journal of Psychiatry, 162, 72-79.

Cotter, D. \& Pariante, C. M. (2002) Stress and the progression of the developmental hypothesis of schizophrenia. British Journal of Psychiatry, I8I, 363-365.

Doraiswamy, P. M., Krishnan, K. R., Figiel, G. S., et al (1990) A brain magnetic resonance imaging study of pituitary gland morphology in anorexia nervosa and bulimia. Biological Psychiatry, 28, 110-116.

Elster, A. D. (1993) Modern imaging of the pituitary. Radiology, 187, I-14 
Eritaia, J., Wood, S. J., Stuart, G.W., et al (2000) An optimized method for estimating intracranial volume from magnetic resonance images. Magnetic Resonance Medicine, 44, 973-977.

First, M., Spitzer, R., Gibbon, M., et al (1997) Structured Clinical Interview for DSM-IV Axis I Disorders. Washington, DC: American Psychiatric Press.

Halbreich, U. \& Kahn, L. S. (2003) Hormonal aspects of schizophrenias: an overview.

Psychoneuroendocrinology, 28 (suppl. 2), I-16.

Krishnan, K. R., Doraiswamy, P. M., Lurie, S. N., et al (1991) Pituitary size in depression. Journal of Clinical Endocrinology and Metabolism, 72, 256-259.

Lum, C., Kucharczyk, W., Montanera, W., et al (2002) The sella turcica and parasellar region. In Magnetic Resonance Imaging of the Brain and Spine (ed. S.W. Atlas), pp. 1283-1362. Philadelphia, PA: Lippincott Williams \& Wilkins.

Lurie, S. N., Doraiswamy, P. M., Husain, M. M., et al (1990) In vivo assessment of pituitary gland volume with magnetic resonance imaging: the effect of age. Journal of Clinical Endocrinology and Metabolism, 7I, 505-508.

McGorry, P. D., Kaplan, I., Dossestor, C., et al (1989) Royal Park Multidiagnostic Instrument for Psychosis. Canberra: National Health and Medical Research Council.

McGorry, P. D., Edwards, J., Mihalopoulos, C., et al (1996) EPPIC: an evolving system of early detection and optimal management. Schizophrenia Bulletin, 22, 305-326.

Mineura, K., Goto, T., Yoneya, M., et al (1987) Pituitary enlargement associated with Addison's disease. Clinical Radiology, 38, 435-437.

Muller, M. B., Lucassen, P. J., Yassouridis, A., et al (200I) Neither major depression nor glucocorticoid treatment affects the cellular integrity of the human hippocampus. European Journal of Neuroscience, 14 $1603-1612$

Myin-Germeys, I., van Os, J., Schwartz, J. E., et al (200I) Emotional reactivity to daily life stress in psychosis. Archives of General Psychiatry, 58, II37-1I44.

Pariante, C. M. (2003) Depression, stress and the adrenal axis. Journal of Neuroendocrinology, 15, 8II-8I2.

Pariante, C. M. \& Miller, A. H. (200I) Glucocorticoid receptors in major depression: relevance to pathophysiology and treatment. Biological Psychiatry, 49 $391-404$

Raison, C. L. \& Miller, A. H. (2003) When not enough is too much: the role of insufficient glucocorticoid signaling in the pathophysiology of stress-related disorders. American Journal of Psychiatry, 160 $1554-1565$

Ryan, M. C., Collins, P. \& Thakore, J. H. (2003) Impaired fasting glucose tolerance in first-episode, drug naive patients with schizophrenia. American Journal of Psychiatry, 160, 284-289.

Sachar, E. J., Kanter, S. S., Buie, D., et al (1970) Psychoendocrinology of ego disintegration. American Journal of Psychiatry, 126, 1067-1078.

Saiardi, A., Bozzi, Y., Baik, J. H., et al (1997)

Antiproliferative role of dopamine: loss of D2 receptors

\section{CLINICAL IMPLICATIONS}

- The first episode of a psychotic disorder is associated with enlargement of the pituitary. One possible interpretation of this finding is that it indicates activation of the hormonal stress response, and especially of the hypothalamic-pituitary-adrenal axis.

- The finding of a reduced pituitary volume in people with established psychosis is similar to that described in people with bipolar disorders and anorexia, and suggests that common pathological mechanisms are present in chronic mental disorders, leading to hypoplasia of the pituitary gland.

- Prospective studies will clarify whether measurements of the stress response can be used to predict the development of psychosis.

\section{LIMITATIONS}

- Because the participants in the first-episode group were receiving antipsychotics, we cannot exclude that pituitary hyperplasia could be related, at least in part, to increased function of prolactin-producing cells activated by neuroleptic treatment.

- It is difficult to distinguish between anterior and posterior pituitary volume using imaging methods.

Without measurements of hormonal levels, our proposed relationship between pituitary hyperplasia and hyperproduction of pituitary hormones remains speculative.

CARMINE M. PARIANTE, MRCPsych, PhD, Division of Psychological Medicine, Institute of Psychiatry, King's College London, UK; KONSTANTINA VASSILOPOULOU, MD, Department of Psychiatry, Eginition Hospital, University of Athens, Greece; DENNIS VELAKOULIS, FRANZCP, Melbourne Neuropsychiatry Centre, Department of Psychiatry, University of Melbourne, Australia; LISA PHILLIPS, MPsych(Clin), Personal Assessment and Crisis Evaluation (PACE) Clinic, Orygen Research Centre, Australia; BRIDGET SOULSBY, MSc, Melbourne Neuropsychiatry Centre, Department of Psychiatry, University of Melbourne, Australia; STEPHEN J.WOOD, PhD, Melbourne Neuropsychiatry Centre, Department of Psychiatry, University of Melbourne, and Brain Research Institute, Melbourne, Australia; WARRICK BREWER, PhD, DEIDRE J. SMITH, GradDipAdol Psych, Orygen Research Centre, Australia; PAOLA DAZZAN, MSc, MRCPsych, Division of Psychological Medicine, Institute of Psychiatry, King's College London, UK; ALISON R. YUNG, FRANZCP, Personal Assessment and Evaluation (PACE) Clinic, Orygen Research Centre, Australia; IOANNIS M. ZERVAS, MD, GEORGE N. CHRISTODOULOU, MD, Department of Psychiatry, Eginition Hospital, University of Athens, Greece; ROBIN MURRAY, FRCPsych, Division of Psychological Medicine, Institute of Psychiatry, King's College London, UK; PATRICK D. McGORRY, FRANZCP, Early Psychosis Prevention and Intervention Centre (EPPIC), Orygen Research Centre, Australia; CHRISTOS PANTELIS, MRCPsych, FRANZCP, Melbourne Neuropsychiatry Centre, Department of Psychiatry, University of Melbourne, and Centre for Neuroscience, University of Melbourne, Australia

Correspondence: Dr Carmine M. Pariante, Division of Psychological Medicine, Box PO5I, Institute of Psychiatry, King's College London, I Windsor Walk, Denmark Hill, London SE5 8AF, UK. Tel: +44 (0)20 7848 0807; fax: +44 (0)207848 005I; e-mail: spjucmp@iop.kcl.ac.uk

(First received 6 August 2003, final revision 8 December 2003, accepted 24 January 2004)

causes hormonal dysfunction and pituitary hyperplasia. Neuron, 19, 115-126.

Sassi, R. B., Nicoletti, M., Brambilla, P., et al (200I) Decreased pituitary volume in patients with bipolar disorder. Biological Psychiatry, 50, 27I-280.

Tandon, R., Mazzara, C., DeQuardo, J., et al (1991) Dexamethasone suppression test in schizophrenia: relationship to symptomatology, ventricular enlargement, and outcome. Biological Psychiatry, 29, 953-964.

Velakoulis, D., Pantelis, C., McGorry, P. D., et al (1999) Hippocampal volume in first-episode psychoses and chronic schizophrenia: a high-resolution magnetic resonance imaging study. Archives of General Psychiatry, 56, $133-141$. 